\title{
Application of D'Arcy Thompson's coordinate transformation approach to clinical genetics photographs using image processing techniques
}

\author{
John H DiLiberti
}

\begin{abstract}
The coordinate transformation approach outlined by D'Arcy Thompson for analysing biological shape was extended using modern computerised image processing techniques so that it could be applied to photographs for the study of patients with syndromes of altered facial morphogenesis. Photographs digitised at a resolution of 512 by $\mathbf{4 8 0}$ pixels were subjected to 'rubbersheeting' transformations using a fast microcomputer. Starting with a photograph of a normal child, a single application of the rubbersheeting algorithm produced features such as an upturned nose quite simply. Other facial anomalies may also be recreated with multiple applications. Preliminary results suggest that this technique may be a useful tool in attempts to understand and analyse the changes in facial configuration in a variety of syndromes with facial anomalies.
\end{abstract}

Evidence of attempts to understand and analyse the geometrical relationships of the various parts of the face may be found in art publications at least as far back as the work of Dürer, ${ }^{1}$ approximately four centuries ago, and perhaps earlier efforts may be identified. During the present century these efforts have shifted towards more scientific goals, as in the work of Thompson, ${ }^{1}$ Bookstein, ${ }^{2}$ Clarren et al, ${ }^{3}$ DiLiberti, ${ }^{4}$ and others. A common theme in some of these approaches has been the transformation of one facial shape to another by altering the scale of representation for all or part of one coordinate axis versus the other. Early use of these coordinate

Medical Computing Research Laboratory, Department of Pediatrics, Saint Francis Hospital and Medical Center, 114 Woodland Street, Hartford, CT 06105-1299, USA, and Division of Medical Genetics, Department of Pediatrics, University of Connecticut Health Center, Farmington, CT, USA.

J H DiLiberti

Received for publication 17 December 1990

Accepted for publication 16 January 1991. transformation techniques was limited because of their mathematically laborious nature. Thompson ${ }^{1}$ does not appear to have used more than, at best, a semiquantitative approach. The advent of modern digital computer hardware has reduced the numerical calculation issues to the point where they are no longer a significant obstacle to implementation. The limitation of the original coordinate transformation approach to line drawings such as outlines or sketches is a significant handicap in the clinical genetics setting since photographs are used routinely to record facial configuration information. Although inherently two dimensional, a photographic image contains three dimensional information by virtue of reflectance characteristics resulting from surface orientation and texture. The ability to apply a more generalised coordinate transformation technique to clinical photographs could therefore be useful as a method of understanding differences in facial configuration. Modern computer image processing methods, in fact, include approaches applicable to photographic images which are equivalent to coordinate transformation. The implementation and application of computerised image processing for the understanding of facial configuration and shape in clinical genetics is described below.

\section{Theoretical considerations}

The concept of the Cartesian coordinate system is certainly familiar to anyone working in a scientific field. Generally, each axis is a linear function of whichever unit of measurement is appropriate. Less commonly, non-linear functions such as logarithmic or exponential scales are used. Usually, the linear, exponential, or logarithmic function is applied uniformly along the entire axis selected. Although generally the most convenient and useful approach, the application of a uniform, analytic function along each axis is by no means mathematically essential. In fact, quite arbitrary numerical coordinate schemes may be used effectively, depending on the application. A shape plotted using $\mathrm{X}$ and $\mathrm{Y}$ coordinates with equal, linear scales will be altered if one or both coordinate axes are transformed. If the $\mathrm{X}$ axis scale is 
uniformly expanded by a factor of 2 , a curve which was originally a square will become a rectangle. Other shapes will be similarly stretched along this axis. If non-linear transformations are applied, or if any transformation is applied to arbitrary regions on either or both coordinate axes, the results may be far more complex. ${ }^{5}$

Dürer's insight that the appearance of the facial outline could be markedly altered in a meaningful way by localised changes in the scales used for the coordinate axes suggested the possibility that coordinate axis transformations might have applicability to the study of biological systems. Thompson ${ }^{1}$ subsequently described this approach in his well known monograph. Dürer and Thompson applied coordinate transformations to outlines or sketches of faces, animals, or biological structures in an effort to understand their shapes (fig 1). Clinical geneticists often work with two dimensional black and white photographs which contain considerably more information than outlines. Consequently, the simple coordinate transformations using traditional plotting techniques which Dürer and Thompson demonstrated are not immediately applicable to photographs. More recently, however, computerised image processing methods have been developed which allow virtually limitless transformations of photographs. ${ }^{6}$

Image processing generally involves the conversion of an image obtained using either an electronic imaging device, such as a video camera, or photographic device into a form which can be manipulated by a computer. ${ }^{7}$ In essence, the image becomes a mosaic of picture elements (pixels), each with an associated intensity and pair of coordinates. In order to obtain reasonably high image quality, a minimum of about 500 pixels in each dimension should be used. Substantially smaller pixel totals lead to considerable degradation of the image and, as expected, increasing
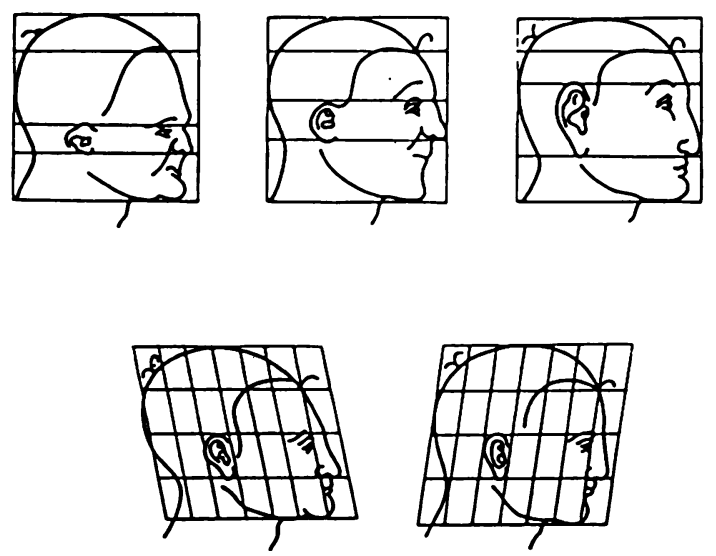

Figure 1 Coordinate transformation of facial caricatures by Dürer (from Thompson ${ }^{I}$ ). the number of pixels in each direction (up to about 2000) will enhance the perceived quality of the image. Pixel counts higher than 2000 will generally not produce a noticeable improvement unless greatly magnified. For comparison, a standard broadcast television image has pixel resolutions of about 300 horizontally by 240 vertically (actually 240 pairs of identical vertical pixels), whereas a high quality photograph will, under optimal conditions, have the equivalent of several thousand pixels of information in each dimension. Each pixel has a numerical intensity corresponding to the brightness at that particular point in the original image. For black and white images the values generally used range from 0 to 255 , whereas for high resolution colour images each primary colour (red, blue, green) will require a separate range as high as 0 to $255^{6}$

Once an image is available in digital form a wide variety of techniques may be applied to enhance, analyse, or modify it. The operations of particular interest in the context of coordinate transformation are geometrical in nature. ${ }^{5}$ To avoid addressing the mathematical issues these are perhaps best visualised by imagining a photograph printed on an elastic sheet. If opposite ends of the sheet are stretched the image is expanded in this axis proportionate to the amount of deformation of the elastic sheet. If the sheet is stretched so that the original dimension is doubled, the result is comparable to the coordinate transformation by a factor of 2 described above. Operations in this mode are still constrained by the fact that we are transforming the image along an entire axis. Although this worked to some extent for Dürer and Thompson when applied to outline sketches, it is a rather crude approach when applied to photographs. A more general technique involves the selection of a single point and the ability to transform the coordinates for a surrounding region of arbitrary size in any direction to any extent. Returning to the elastic photograph model, this new technique may be visualised as the ability to select any point, attach a handle behind it, and pull it in any direction. Sometimes called 'rubbersheeting' for obvious reasons, this method may be applied repetitively to an image to achieve the desired results. In the rubbersheet model, after each successive application the image would be rephotographed and applied to a new sheet, preserving the new relationships in the image.

\section{Materials and methods}

Black and white negatives or colour slides were digitised at a resolution of 512 by 480 pixels with 256 shades of grey using an Imaging Technology PC Vision Plus Board. Image processing was done on a PSI $33 \mathrm{MHz}$ Intel 386 based computer equipped with 16 megabytes of RAM and a Weitek 3167 numerical coprocessor. Images were viewed and manipulated 
using a Mitsubishi $48 \mathrm{~cm}$ colour monitor with a resolution of 1024 by 768 pixels driven by a Number Nine Computer Revolution graphics board. Software was written in the $C$ programming language and

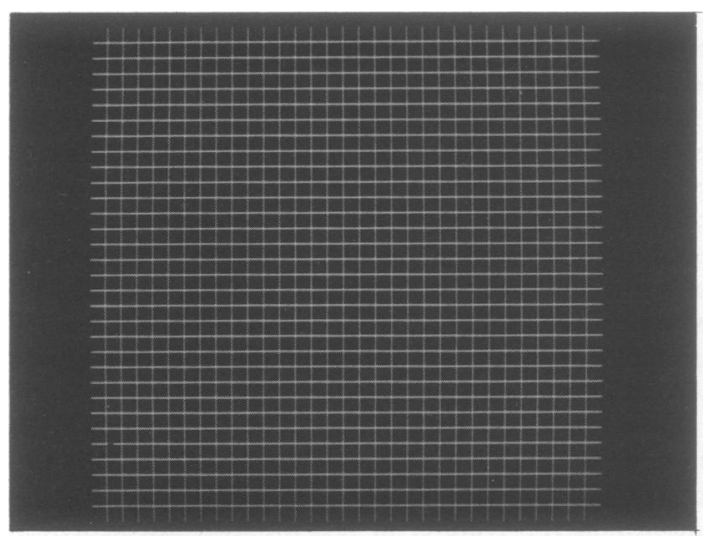

Figure 2 Square grid on computer monitor.

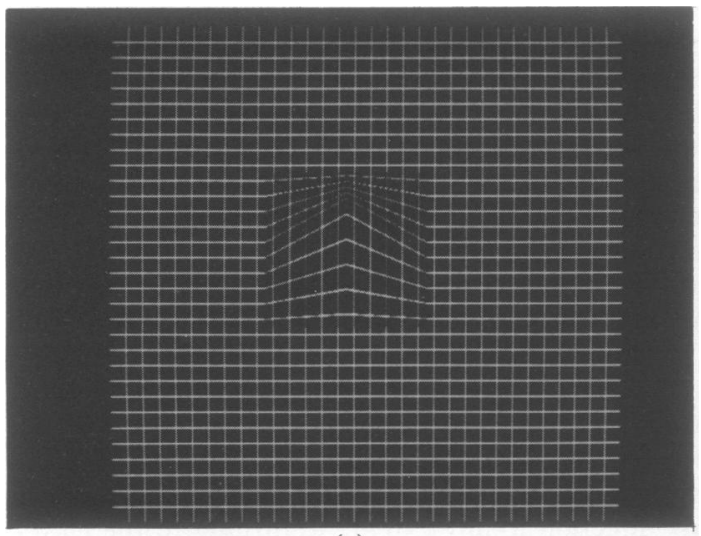

(a)

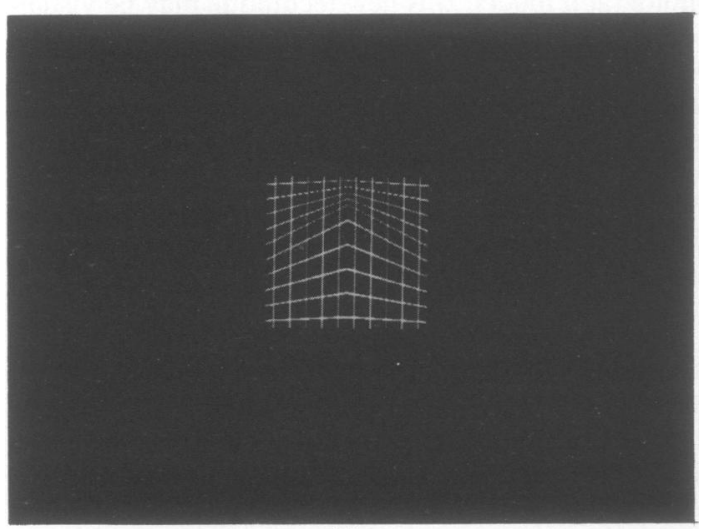

(b)

Figure 3 (a) Warped square grid and (b) warped area alone in square grid. compiled with the NDP C compiler and linked with the Phar Lap DOS extender.

The software was designed to perform repetitive rubbersheeting operations under the control of the operator. A rectangular region of interest is delimited, generally symmetrically arranged about the point on the photograph to be moved. Once this point has been selected, the operator may then choose the position within the constraining box to which it will be warped. Since the computer memory image is a mosaic of pixel information, the rubbersheeting operation will, in general, produce empty pixels in stretched areas and extra pixels in compressed areas. This problem was avoided using the techniques of output to input mapping and bilinear interpolation. ${ }^{6} 8$

Rubbersheeting was applied to several computer generated geometrical figures, including a rectilinear grid and circle, along with an image of a normal infant. Because normal facial configuration is so varied, it seemed that attempting to transform a normal configuration into a syndromic form would be a more instructive starting point, since moving from general to specific is conceptually simpler.

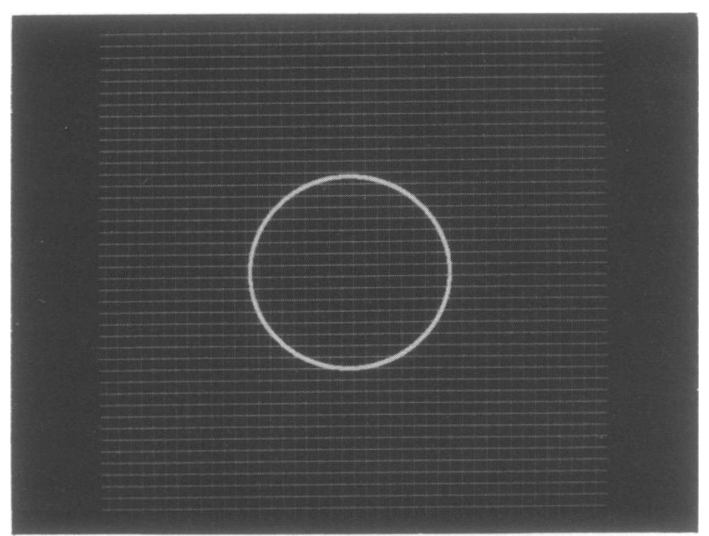

Figure 4 Circle superimposed on quadrilateral array.

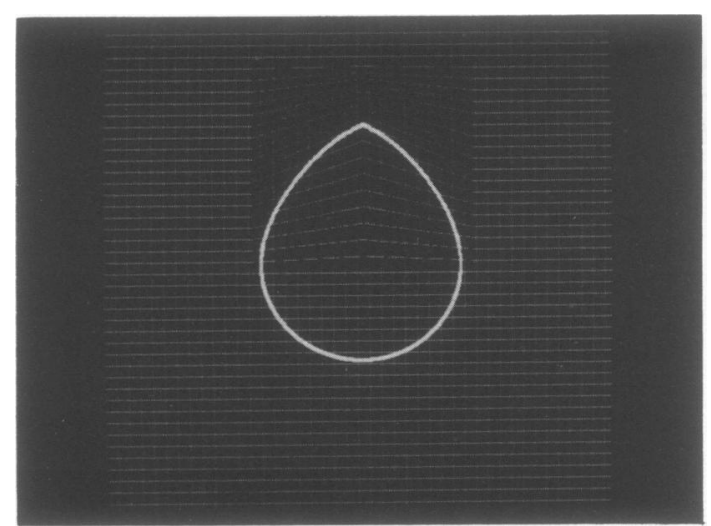

Figure 5 Single warping of circle. 


\section{Results}

A single application of the rubbersheeting technique to a square grid is illustrated in figs 2 and 3 , whereas figs 4,5 , and 6 show the sequential application to a circle. Figs $7,8,9$, and 10 show single and multiple

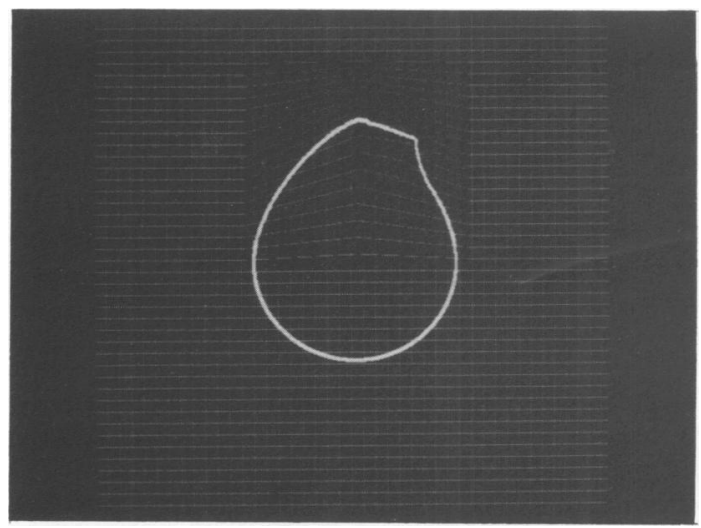

Figure 6 Double warping of circle.
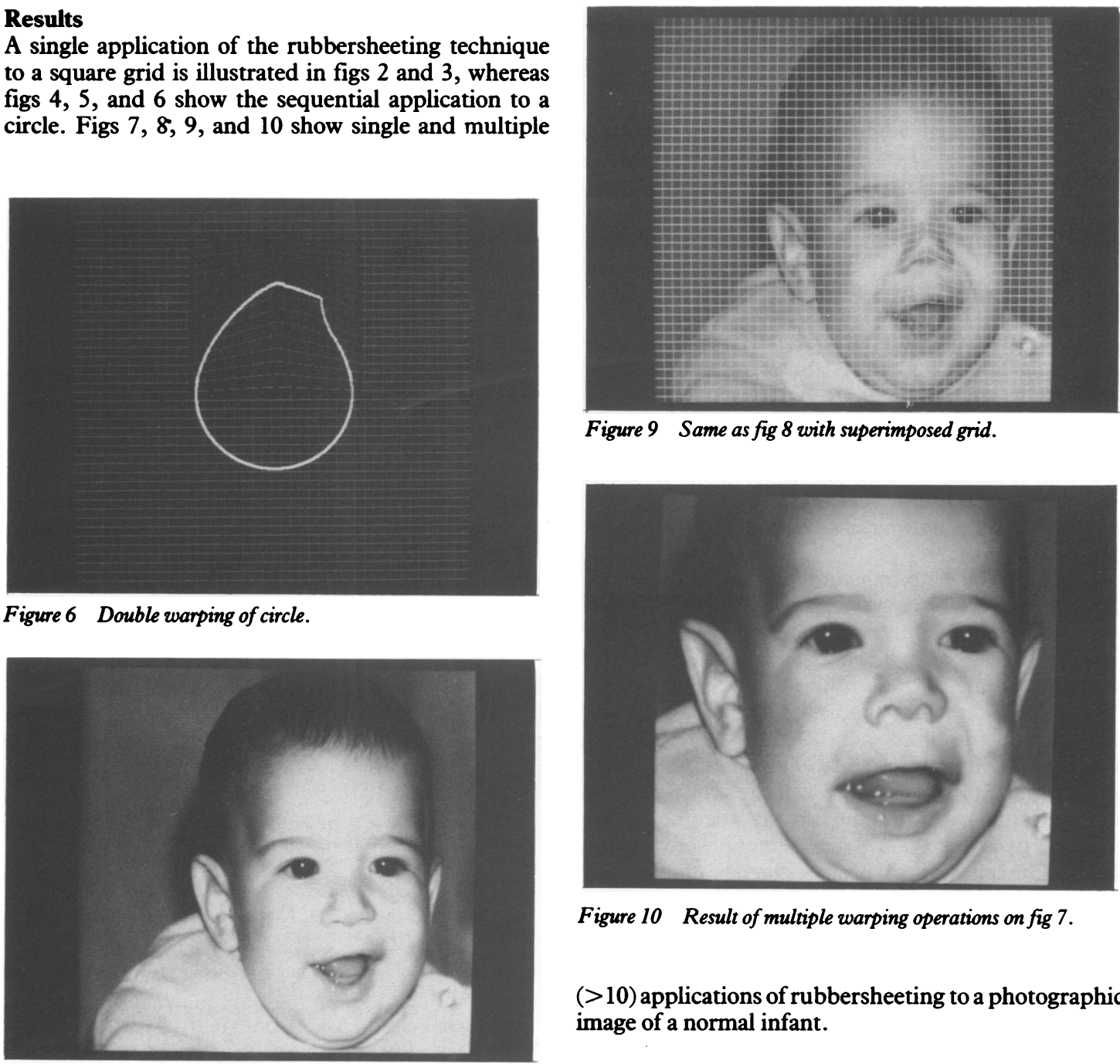

Figure 9 Same as fig 8 with superimposed grid.

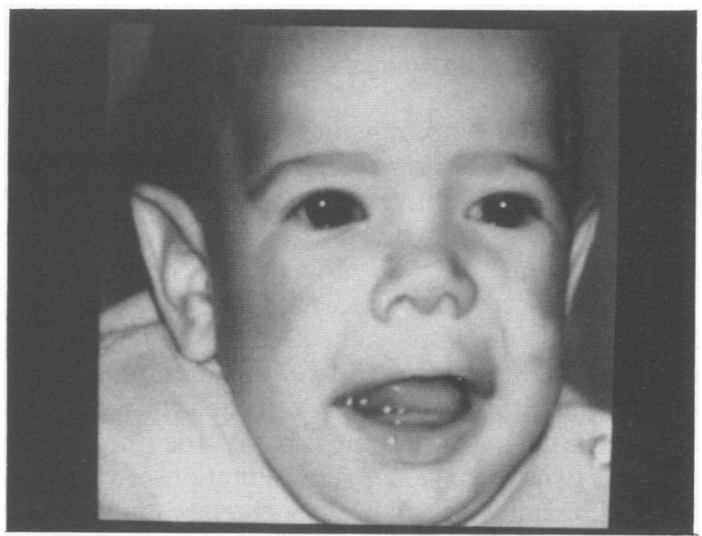

Figure 10 Result of multiple warping operations on fig 7.

(>10) applications of rubbersheeting to a photographic image of a normal infant.

Figure 7 Digitised photograph of normal infant.

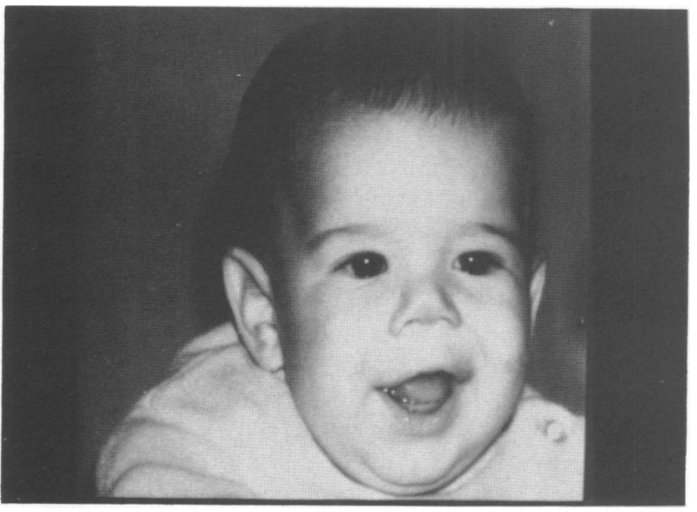

Figure 8 Single warping to create upturned nose.

\section{Discussion}

The transformation of the rectangular grid (fig 3) illustrates nicely the fundamental effects of rubbersheeting. The delimited region stands out clearly from the area which has not been altered by the process. The amount of deformation is greatest in the immediate proximity of the selected point and drops off as the delimited boundaries are approached. Since the selected point was moved in a direction parallel to one axis of the grid, none of the points involved in the deformation had changes in coordinates along the perpendicular axis, hence the vertical lines all remain vertical and parallel while the horizontal lines have been stretched and become angulated. A similar result has occurred with the single application of rubbersheeting to the circle, leading to an ovoid shape. When a second operation was done at a diagonal 
orientation the result is more complex, as seen in fig 6.

When applied to an actual photograph of a normal infant the results are more interesting. Figs 8 and 9 show the effect of a single rubbersheeting operation which moved a point on the tip of the nose upwards, with and without a superimposed grid, which indicates the extent and range of deformation. As can be seen, a single application of this image processing method convincingly transforms a nose of normal appearance into an upturned nose comparable to one seen in a variety of malformation syndromes. Other facial features may also be altered from normal appearing to syndromic appearing forms following one or more applications of this method. Fig 10 shows the first attempt at using this technique to modify a normal infant face to create a 'syndromic' appearance. An operator with more experience in drawing would probably be far more successful at transforming a normal face into an image recognisable as a specific syndrome.

While the ability to transform an image of a normal face into one which mimics a known syndrome may seem at first to be only a laboratory curiosity, there are several possible applications, some which may not be immediately obvious. First, in the process of attempting to create a syndromic facial appearance, the operator develops considerable understanding regarding the important and subtle alterations which constitute the differences between the syndromic and normal features. Despite the fact that the photograph is only a two dimensional representation of the face, the shading and textural aspects give the operator the feeling of modelling in nearly a full three dimensions. Thus, rubbersheeting may be a good tool to aid clinical geneticists in the more objective description of syndromes. By actually transforming facial features in images, it appears to be much easier to identify which aspects are most characteristic or necessary to give the impression of a specific syndrome.

The second, and potentially very powerful, way rubbersheeting may be applied to clinical genetics is an extension of the first application noted above. If a skilled operator of the rubbersheeting program can consistently produce images which mimic known syndromes, then the sequence of rubbersheeting operations presumably contains the information which objectively differentiates a normal face from that found in a specific syndrome. The program could fairly easily be enhanced to keep track of this sequence of steps and the attendant numerical parameters. Careful analysis of these data could lead to considerably better understanding of the alterations of facial structures in malformation syndromes on at least a qualitative, but perhaps quantitative, basis.

Eventually, as three dimensional facial image data are routinely collected on clinical genetics patients, a variation of rubbersheeting could be extended to make this an even more powerful technique. If a representation of the surface of the face were projected on a video monitor and the operator, using elastic deformations, could create an image of a specific syndrome, then the steps outlined above, identifying and analysing a sequence of operations, may provide a basis for obtaining objective information about the facial shape differences which exist in various syndromes.

This work was presented, in part, at the David W Smith Workshop on Malformations and Morphogenesis, Lexington, Kentucky, August 1990.

1 Thompson DW. On growth and form. Cambridge: Cambridge University Press, 1961:268-325.

2 Bookstein FL. The measurement of biological shape and shape change. Lecture Notes in Biomathematics. Volume 24. Berlin: Springer-Verlag, 1978.

3 Clarren SK, Sampson PD, Larsen J, et al. Facial effects of fetal alcohol exposure: assessment of photographs and morphometric analysis. Am F Med Genet 1987;26:651-66.

4 Diliberti JH. Use of computers in dysmorphology. 7 Med Genet 1988:25:445-53.

5 Penna MA, Patterson RR. Projective geometry and its applications to computer graphics. Engelwood Cliffs, NJ: Prentice-Hall, 1986.

6 Niblack W. An introduction to digital image processing. Englewood Cliffs, NJ: Prentice-Hall International, 1986.

7 Pavlidis T. Algorithms for graphics and image processing. Rockville, MD: Computer Science Press, 1982:27-46.

8 Castleman KR. Digital image processing. Englewood Cliffs, NJ: Prentice-Hall, 1979:113-7. 